\title{
Vibration Fault Diagnosis of Hydroelectric Unit Based on LS-SVM and Information Fusion Technology
}

\author{
Qiguo YAO ${ }^{a,}{ }^{*}$, Yuliang LIU $^{b}$ \\ School of Naval Architecture \& Mechanical-electrical Engineering, Zhejiang Ocean University, \\ Zhoushan, Zhejiang, 316022, China \\ ayaoqiguo@163.com, blyl_zjou@126.com, "corresponding author
}

Keywords: Hydroelectric Unit; Vibration; Fault Diagnosis; Support Vector Machine; Information Fusion

\begin{abstract}
Vibration fault diagnosis of hydroelectric unit was investigated using method of least square support vector machine (LS-SVM) and Dempster-Shafer theory (D-S Theory). Spectrum and amplitude characteristic was acted as eigenvector of learning samples to train the constructed LS-SVM regression and classifier for realizing mapping relationship between the fault and the characteristic. Information fusion was realized after completing local diagnosis, and then fault diagnosis was achieved. Experiments show that the method has a rapidly diagnostic process and generalization performances. It is suitable for the vibration fault diagnosis of hydroelectric unit.
\end{abstract}

\section{Introduction}

As effective tool solving complicated non-linear problem, artificial neural network has been used widely in diagnosing equipment faults field [1], and is another artificial intelligence technology applied to hydroelectric unit fault diagnosis field following expert system [2]. But the neural network technology is only a kind of heuristic technology depending on experience, lacking the solid theoretical foundation. Its learning process adopts the empirical risk minimization principle, and it is apt to fall into the local minimum and is of weak generalization ability.

In addition, the complexity of its algorithm is affected greatly by complexity of network structure and sample complexity, and these shortcomings have already further restricted the application and development of the artificial neural network in the intellectual fault diagnosis field. The support vector machine (SVM) proposed by $\mathrm{V}$. Vapnik has received more and more attention in recent years. SVM is a new machine learning method aimed at small samples, on the basis of statistics learning theory and structural risk minimization principle. It is of terse mathematical operator, intuitionistic geometry, outstanding study performance and popularizing ability. It can overcome the above-mentioned defects of the neural network and has gained widely application in pattern recognition, regression analysis and function approximation [3].

In order to improve SVM calculation speed, the least square support vector machine (LS-SVM) scheme has been proposed by Suykens et al on the basis of standard SVM [4]. SVM have the advantages of quick calculation speed and strong generalization ability, and Dempster-Shafer theory (D-S theory) [5] has better disposing ability to uncertain information. In order to improve diagnosis reliability and accuracy, in this paper a classified and open fault diagnosis model for Hydro-turbine Generating Unit is put forward based on SVM and D-S theory and a classifier, and regression set is constructed based on LS-SVM.

\section{Classification and Regression Theory of LS-SVM}

Standard SVM. The main idea of SVM datum classification is t o map a data sample to a $R^{n}$ imensions (dimensions perhaps very high) high character space from originally space $R^{m}$ dimensions by using a nonlinear mapping $\varphi$, then to divide the dots of training samples by optimal hyperplane in high dimensions character space, and to make the dots far from the optimal hyperplane as possible. 
The nonlinear classification algorithm is as follows [5]:

For given samples $\left(x_{i}, x_{j}\right), \quad i=1,2, \ldots, N, x_{i} \in R^{m}, m$ is the $i^{\text {th }}$ input pattern, $y_{i} \in(+1,-1)$ is the correspondingly desired output. Using a nonlinear mapping $\phi(x)=\left[\phi_{1}(x), \phi_{2}(x), \ldots \phi_{N}(x)\right]^{\mathrm{T}}$ to map the input datum to $N$ imensions character space from originally space, and construct optimal classification hyperplane

$\sum_{i=1}^{N} w_{i} \phi_{i}(x)+b=0$

Where, $w$ is a new weight value vector which connects the character space and output space, and $b$ is threshold value. They need to be evaluated before SVM is designed. Considering error in datum classification in high dimensions, SVM introduces slack variable $\xi_{j} \geq 0$ (reflects the distance between actual indicated value and SVM output). Then the matter is minimization $\|w\|$ while satisfying constraint,

$y_{i}\left[\sum_{i=1}^{N} w_{i} \phi_{i}(x)+b\right] \geq 1-\xi_{i}$

And its standard form is

$\min \quad J=\frac{1}{2} w^{\mathrm{T}} w+\gamma \sum_{i=1}^{N} \xi_{i}$

s.t. $\quad y_{i}\left[\sum_{i=1}^{N} w_{i} \phi_{i}(x)+b\right] \geq 1-\xi_{i}, \quad \xi_{i} \geq 0$

Where, $\gamma$ is penalty degree while beyond control error of samples.

The solution of this convex quadratic programming can be solved by solving its dual problem, i.e:

$\max \quad Q(\alpha)=\sum_{i=1}^{N} \alpha_{i}-\frac{1}{2} \sum_{i=1}^{N} \alpha_{i} \alpha_{j} y_{i} y_{j}\left[\phi\left(x_{i}\right) \cdot \phi\left(x_{j}\right)\right]$

s.t. $\quad \sum_{i=1}^{N} \alpha_{i} y_{i}=0, \quad \alpha_{i} \geq 0$

SVM uses kernel function to replace the dot product $\phi\left(x_{i}\right) \cdot \phi\left(x_{j}\right)$ rather than to solve it directly in high dimensions character space. According to functional theory, the dot product $\phi\left(x_{i}\right) \cdot \phi\left(x_{j}\right)$ in high dimensions can be replaced by using a kernel function $K\left(x_{i}, x_{j}\right)=\phi\left(x_{i}\right) \cdot \phi\left(x_{j}\right)$ that meets mercer condition in input space, and the concrete form of nonlinear mapping is not necessary (away from the dimension avalanche).

LS-SVM classification (SVC). Training standard SVM is required solve convex quadratic programming, and the optimal solution is exclusive. But its algorithm complexity depends on the number of samples. The sample is the bigger in data amount, the slower the computational speed is, the greater the memory demand is. Considering function complexity and fitting errors, and according to structural risk minimization principle, LS-SVM is a redefining optimization by use of changing constrained condition (3) of standard SVM into equality and cost function [6]. That is:

$\min \frac{1}{2} w^{\mathrm{T}} w+\gamma \frac{1}{2} \xi_{i}^{2}$

s.t. $\quad y_{i} \cdot\left[\sum_{i=1}^{N} w_{i} \phi_{i}(x)+b\right]=1-\xi_{i}, \quad \xi_{i} \geq 0$

For optimal formula (4), drawn into Lagrange function, the follow equation can be obtained.

$L=\frac{1}{2} w^{\mathrm{T}} w+\gamma \frac{1}{2} \sum_{i=1}^{l} \xi_{i}^{2}-\sum_{i=1}^{l} \alpha_{i}\left\{y_{i}\left[w^{\mathrm{T}} \phi\left(x_{i}\right)+b\right]\right\}-1+\xi_{i}$

Where, $\alpha_{i}$ is Lagrange multiplier. Solving $L$ partial derivative to $w, \xi, b$ and $\alpha_{i}$, then zero setting. A new formula is as follows. 


$$
\begin{aligned}
& w=\sum_{i=1}^{N} \alpha_{i} y_{i} \phi\left(x_{i}\right) \\
& \sum_{i=1}^{N} \alpha_{i} y_{i}=0 \\
& \alpha_{i}=\gamma_{i} \\
& y_{i}\left[w^{\mathrm{T}} \phi\left(x_{i}\right)+b\right]-1+\xi_{i}=0, \quad(i=1,2, \ldots, N)
\end{aligned}
$$

Based on Karush-Kuhn-Tucker (KKT) condition, combining these formula and elimination $w, \xi$, then a linear equation can be obtained as follows.

$$
\left[\begin{array}{cc}
0 & Y^{\mathrm{T}} \\
Y & \Omega+\gamma^{-1} I
\end{array}\right]\left[\begin{array}{l}
b \\
\alpha
\end{array}\right]=\left[\begin{array}{c}
0 \\
1_{v}
\end{array}\right]
$$

Where, $\Omega=y_{i} y_{j} \phi\left(x_{i}\right)^{T} \cdot \phi\left(x_{j}\right)=y_{i} y_{j} K\left(x_{i}, x_{j}\right), Y=\left[y_{1}, y_{2}, \ldots y_{n}\right], 1_{v}=[1,1, \ldots, 1]$.

The optimization of standard SVM is transformed into solving above-mentioned linear equation. This linear equation can be solved by least square method, simplify computational complexity. Therefore, LS-SVM has a faster training speed than standard SVM.

LS-SVM regression (SVR). The solving method of SVM can be applied to solve regression problem and the main character of maximum interval algorithm can be hold. But the regression of LS-SVM adopts least square regression (ridge regression). As the classification algorithm, LS-SVM regression also is an optimization by changing the inequality constrained condition (3) of standard SVM into equality and cost function [7].

$\min \frac{1}{2} w^{\mathrm{T}} w+\gamma \frac{1}{2} \xi_{i}^{2}$

s.t. $\quad y_{i}=w^{\mathrm{T}} \phi_{i}(x)+b+\xi_{i}, \quad \xi_{i} \geq 0$

Similar the algorithm 1.2, the solution of LS-SVM regression can be obtained by solving linear equations (13):

$$
\left[\begin{array}{cc}
0 & 1_{v}{ }^{\mathrm{T}} \\
1_{v} & \Omega+\gamma^{-1} I
\end{array}\right]\left[\begin{array}{l}
b \\
\alpha
\end{array}\right]=\left[\begin{array}{l}
0 \\
Y
\end{array}\right]
$$

\section{Fault Diagnosis System Model}

Fault character space is decomposed into different character subspaces, i.e. fault character space is decomposed into vibration frequency spectrum and amplitude spectrum, and the whole fault space is decomposed into generator fault subspace, rotator bearing fault subspace and flow passage component subspace. Every character space and fault space adopt respectively a LS-SVR to carry out a mapping from each character subspace to fault space, and to achieve initial diagnosis for fault character layer. And then design a LS-SVC to carry out decision diagnosis classification after initial diagnosis fusion.

The reasons of selecting LS-SVR are that the real value of initial diagnosis output has a meaning of denoting current fault classification accuracy, in generally, the real value is bigger and the classification result is more credible; and if only using 0 and 1 two valued vector as input of the SVM final decision, assuring to obtain a good generalization of SVM out is very difficult.

The essential of information fusion is a process that different evidence composes new evidence in the same frame. The scheme diagram of fault diagnosis system model of hydropower generating unit is given in Fig 1. 


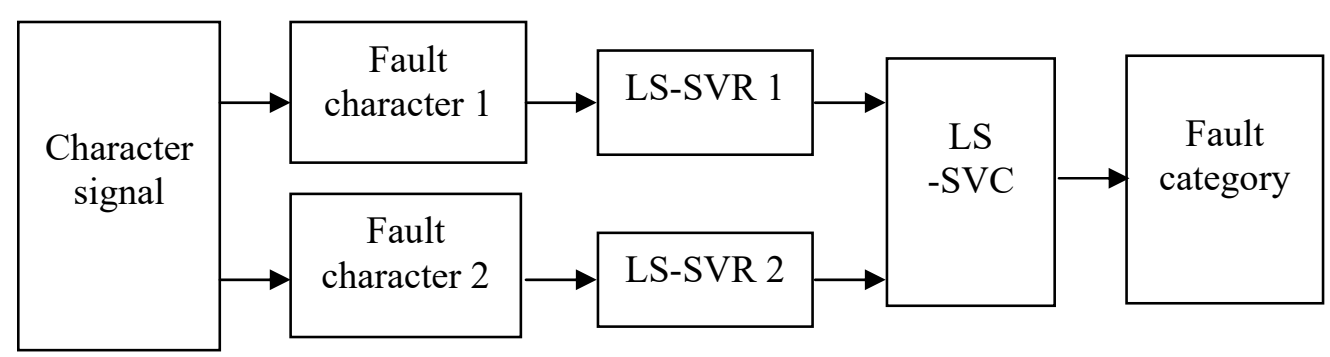

Fig. 1 Fault diagnosis system model of hydroelectric unit

The diagnosis process of fault diagnosis system model based on LS-SVM and information fusion scheme of hydropower generating unit is as follows.

(1)To ready training set and test set, using learning sample of two character spaces to training each SVR; (2)Fusion result of SVR output is as input of SVM to train SVC; (3)The diagnosis system is tested by using test sets.

\section{Case Analysis of Fusion Diagnosis}

Diagnosis case. In order to validate the availability of fusion diagnosis scheme based LS-SVM and $D-S$ theory, the 5 familiar faults of hydropower generating unit i.e. unbalance of rotator, eccentricity of rotator, movement collision, vortex of draft tube and Karman vortices, are introduced as a fault diagnosis domain, and compose identification frame $\Theta=\left\{F_{1}, F_{2}, F_{3}, F_{4}, F_{5}\right\}$ of $D-S$ theory. On the basis of fault mechanism and fault symptom, two local diagnostic SVR of vibration frequency spectrum and vibration amplitude are selected. In vibration frequency spectrum SVR, using five fault symptom parameters of $(0.18 \sim 0.2) f_{0},(1 / 6 \sim 1 / 2) f_{0}, 1 f_{0}, 2 f_{0}$ and $3 f_{0}$ to identify the fault, and $f_{0}$ is rotator frequency. In vibration amplitude SVR, using four relations of vibration amplitude and speed, load, scroll casing pressure, discharge as fault symptom parameter to identify the fault. Table 1 and Table 2 are the standard learning samples of vibration frequency spectrum and vibration amplitude [6].

Table. 1 Samples of vibration frequency spectrum

\begin{tabular}{llllll}
\hline \multicolumn{1}{c}{ Types of fault } & \multicolumn{5}{c}{ Fault characters } \\
\cline { 2 - 6 } & $(0.18 \sim 0.2) f_{0}$ & $(1 / 6 \sim 1 / 2) f_{0}$ & $1 f_{0}$ & $2 f_{0}$ & $3 f_{0}$ \\
\hline$F_{1}$ Unbalance of rotor & 0.01 & 0.12 & 0.93 & 0.02 & 0.20 \\
$F_{2}$ Eccentricity of rotor & 0.01 & 0.03 & 0.68 & 0.98 & 0.82 \\
$F_{3}$ Movement collision & 0.06 & 0.05 & 0.92 & 0.52 & 0.48 \\
$F_{4}$ Vortex draft tube & 0.05 & 0.95 & 0.07 & 0.02 & 0.01 \\
$F_{5}$ Karman vortice & 0.93 & 0.04 & 0.10 & 0.11 & 0.06 \\
\hline
\end{tabular}

The diagnosis system is validated by actual measurement information [8] of some hydropower generating unit. Table 3 and Table 4 are the sampling value (already normalization) of two symptom domains. Table 5 is the local diagnostic results of frequency and amplitude symptoms SVR.

Table. 2 Samples of vibration amplitude

\begin{tabular}{lllll}
\hline \multirow{2}{*}{ Types of fault } & \multicolumn{4}{c}{ Fault characters } \\
\cline { 2 - 5 } & $\begin{array}{l}\text { Vibration and } \\
\text { rotate speed }\end{array}$ & $\begin{array}{l}\text { Vibration } \\
\text { and load }\end{array}$ & $\begin{array}{l}\text { Vibration and } \\
\text { scroll case } \\
\text { pressure }\end{array}$ & $\begin{array}{l}\text { Vibration and } \\
\text { discharge }\end{array}$ \\
\hline$F_{1}$ Unbalance of rotor & 0.97 & 0.17 & 0.02 & 0.01 \\
$F_{2}$ Eccentricity of rotor & 0.97 & 0.95 & 0.52 & 0.45 \\
$F_{3}$ Movement collision & 0.96 & 0.01 & 0.03 & 0.16 \\
$F_{4}$ Vortex draft tube & 0.10 & 0.95 & 0.01 & 0.08 \\
$F_{5}$ Karman vortice & 0.02 & 0.07 & 0.01 & 0.95 \\
\hline
\end{tabular}

After above-mentioned two local diagnostic results are sent to SVC, the diagnostic result of frequency spectrum is unbalance of rotator and the result of vibration amplitude is eccentricity of 
rotator. The obtained conclusions are obviously contradictory. If fusing the two local diagnostic results (fusion result is in Table 6) as an evidence, then the diagnostic result is unbalance of rotator (confidence boosts to 0.8123 ), and is correspond to actual condition. Hence many symptoms information fusion can improve basic probability assignment, the uncertainty of hydropower generating unit decrease, the accuracy of fault diagnosis is improved, and this fully reflects that using fusion information of local LS-SVR output to diagnose is very available.

Table. 3 Test samples of vibration frequency spectrum

\begin{tabular}{lllll}
\hline$(0.18 \sim 0.2) f_{0}$ & $(1 / 6 \sim 1 / 2) f_{0}$ & $1 f_{0}$ & $2 f_{0}$ & $3 f_{0}$ \\
\hline 0.01 & 0.02 & 0.96 & 0.05 & 0.10 \\
\hline
\end{tabular}

Table.4 Test samples of vibration amplitude

\begin{tabular}{llll}
\hline $\begin{array}{l}\text { Vibration and } \\
\text { rotation speed }\end{array}$ & $\begin{array}{l}\text { Vibration and } \\
\text { load }\end{array}$ & $\begin{array}{l}\text { Vibration and scroll } \\
\text { case pressure }\end{array}$ & Vibration and discharge \\
\hline 0.91 & 0.10 & 0.12 & 0.09 \\
\hline
\end{tabular}

Table. 5 Local diagnostic results of frequency and amplitude symptoms SVR

\begin{tabular}{llllll}
\hline Assignment function $m(F)$ & $F_{1}$ & $F_{2}$ & $F_{3}$ & $F_{4}$ & $F_{5}$ \\
\hline Spectrum information & 0.6714 & 0.0738 & 0.0942 & 0.0671 & 0.0935 \\
Amplitude information & 0.3583 & 0.0956 & 0.3855 & 0.0802 & 0.0795 \\
\hline
\end{tabular}

Table. 6 Final diagnosis conclusion

\begin{tabular}{lllll}
\hline$F_{1}$ & $F_{2}$ & $F_{3}$ & $F_{4}$ & $F_{5}$ \\
\hline 0.8123 & 0.1011 & 0.0225 & 0.0472 & 0.0169 \\
\hline
\end{tabular}

Analysis. Influence of Kernel Function and its Parameter on Diagnosis. In order to compare the regression and classification impact of different kernel function, two kernel functions (polynomial kernel and radial basis kernel) are introduced. To select the SVM parameter, the $K$ cross validation mesh search method is adopted, $\varepsilon=0.01$. The result shows that the radial basis kernel $\delta^{2}=[1,3]$ is better. For $\mathrm{d} \leq 3$, the classification and regression effect of polynomial kernel and radial basis kernel is on the same level, but calculation speed of the radial basis kernel is faster than that of the polynomial kernel. Therefore radial basis kernel is introduced.

LS-SVM Compare with Neural Network Model. Using BP network to diagnose test data in order to compare the diagnostic effect of LS-SVM and neural network model. Topological structure of BP network is 5-13-5 and error is 0.001. The result indicates that BP network can diagnose the fault of unbalance of rotator, but its confidence (only 0.66) is lower than the SVM, and this shows that the generalization of BP network is weaker than the SVM.

\section{Conclusion}

By applying LS-SVM to the fault diagnosis of hydropower generating unit, the results showed that the SVM generalization is powerful in small samples, especially to acquire fault samples are difficult in fault diagnosis of hydropower generating unit.

Use SVR to initial diagnosis and then use the initial result to decision fusion based on D-S theory. It can be achieved that the weaker diagnostic decision effects support stronger diagnostic decision, and the diversity problems of actual engineering also can be solved.

The fault diagnosis of hydropower generating unit can be achieved fast and availably owing to the effective combination of LS-SVM and D-S theory.

\section{Acknowledgement}

This study is supported by the project of public welfare of Zhejiang province (Grant No. 2015C31072). 


\section{References}

[1] Heji Yu, Changzheng Chen, Sheng Zhang. Intelligent diagnosis based on neural networks [J]. Journal of vibration engineering, 2000,13(2):202-209.

[2] Xiangqian $\mathrm{Fu}$, Guangling Liu, jin Jiang. Application of BP neural networks to condition monitoring and fault diagnosis system of hydro-generator units[J]. Journal of Wuhan University (Engineering),2002,35(1):24-28.

[3] Rong Jia, Liang Bai, Xingqi Luo, et al. Expert system on fault diagnosis based on neural network for hydropower units[J]. Journal of Hydroelectric Engineering, 2004,23(6);120-123.

[4] Zhousuo Zhang, Lingjun Li, Zhengji He. Research on diagnosis method of machinery fault based on support vector machine [J]. Journal of Xi' an Jiaotong University, 2002,36(12):1303-1306.

[5] Weiwu Yan, Junlin Chang, Huihe Shao. Least square SVM regression method based on sliding time window and its simulation[J]. Journal of Shanghai Jiaotong University, 2004, 38(4):521-524.

[6] Xiaoping Yang, Haipeng Nan, Jiangbin Zhang. Application of information fusion technology on fault diagnosis of hydropower generating unit[J]. Journal of Hydroelectric Engineering, 2004, 23(6): 111-115.

[7]Xingguo Cai, Ping Ma. Study on simultaneous fault diagnosis based information fusion technique[J]. Proceedings of the CSEE, 2004,24(10):238-243.

[8]Daoli Zhao, Wei Ma, Wuke Liang, et al. On data fusion fault diagnosis and simulation of hydro-power units vibration[J]. Proceedings of the CSEE, 2005,25(20):137-142. 